\title{
Evolution et conservation de Trypanosoma cruzi chez Ornitbodorus moubata
}

\author{
par J. LAPIERRE \\ (avec la collaboration technique de M. GiLles) \\ Service de Parasitologie, Mycologie, U.E.R. Cochin-Port-Royal \\ 27, rue du Faubourg-St-Jacques, F. 75 -Paris, $14^{\mathrm{e}}$
}

\begin{abstract}
Résumé
L'étude de l'évolution de Trypanosoma cruzi (souche Tulahuen) dans le tube digestif d'Ornithodorus moubata permet de démontrer :

$1^{\circ}$ Que les formes Trypanosomes métacycliques apparaissent vers le $5^{\circ}$ jour.

$2^{\circ}$ Que l'inoculation du contenu digestif est infectante sans interruption du $1^{\mathrm{er}}$ au $30^{\mathrm{e}}$ jour suivant le repas infectant. Ceci amène à penser que les Trypanosomes ingérés - contrairement à ce qui est généralement admis - conservent leur pouvoir infectant pendant au moins 5 jours (date d'apparition des Trypanosomes métacycliques) et certainement au-delà sous des aspects morphologiques dégradés particuliers, évoquant un enroulement du parasite sur lui-même ou réalisant une forme arrondie avec enroulement intérieur du flagellé. Cet aspect a pu les faire considérer comme une étape leishmania.

$3^{\circ}$ Que la souche de $T$. cruzi (Tulahuen) conserve intégralement son pouvoir pathogène chez $O$. moubata après plus de deux ans (791 jours).

Sur le plan pratique, la commodité d'un tel mode de conservation de souches de $T$. cruzi au laboratoire doit être prise en
\end{abstract}


considération en raison de la facilité d'élevage des Ornithodores et de leurs faibles exigences quant à la fréquence des repas par comparaison avec les Réduvidés. L'écart des repas chez certains Ornithodores a été en effet de cinq mois à un an!

Sur le plan épidémiologique, cette notion permet de retenir l'éventualité d'une transmission de la maladie de Chagas par l'Ornithodore, bien que l'Ornithodore ne puisse être considéré que comme un hôte vicariant.

$4^{\circ}$ Dans certains cas, il a été possible d'obtenir par passage sur l'Ornithodore l'exaltation de la virulence d'une souche de $T$. cruzi au préalable atténuée par culture.

$5^{\circ}$ L'infection à $T$. cruzi se développe très bien simultanément chez l'Ornithodore avec l'infection à Borrelia duttoni.

\section{Summary}

Study of the life cycle of T. cruzi (Tulahuen strain) in the digestive tract of Ornithodorus moubata shows:

$1^{\circ}$ That the metacyclic trypanosomes make their appearance on about the 5 th day.

$2^{\circ}$ That inoculation of the digestive content is infective from the Ist till the 30th day after the infective bite. This leads to think that ingested trypanosomes, contrarily to what is generally admitted, keep their infective ability at least for 5 days (date of apparition of metacyclic trypanosomes) and certainly longer under particular morphological aspects, such as rolled up, rounded forms with twisted flagella which have been considered at that phase as Leishmania forms.

$3^{\circ}$ That the Tulahuen strain of $T$. cruzi keeps its infective power in $O$. moubata longer than 2 years (791 days).

At a practical point of view the easiness of such a technic for keeping strains of $T$. cruzi in the laboratory must be considered: Ornithodorus are easy to breed on account of their poor needs for feeding in comparison with reduvid bugs. Indeed they may be fed every six months, some every years.

At an epidemiological point of view it may be possible to consider the eventual transmission of Chagas disease by Ornithodorus acting as an unusual host.

$4^{\circ}$ In some cases it has been possible to enhance the virulence of a strain of $T$. cruzi which had been reduced by culture.

$5^{\circ}$ Infection with $T$. cruzi developps easily in Ornithodorus infected simultaneously with Borrelia duttoni. 
En dehors des conditions de la transmission naturelle par les Réduvidés (Triatomes et Rhodnius), différents Arthropodes vicariants peuvent être infestés expérimentalement et éventuellement jouer un rôle dans la transmission de Trypanosoma cruzi.

En effet, si Yamasaki (1924), Mayer et Da Rocha Lima (1914) n'ont pas réussi à obtenir l'évolution, respectivement chez la puce du chien et les moustiques (Stegomya fasciata, Culex pipiens), l'ubiquité parasitaire de $T$. cruzi est telle que de nombreux succès ont été enregistrés avec d'autres Arthropodes.

En 1912, E. Brumpt fait évoluer avec facilité Trypanosoma cruzi chez quatre espèces de Punaises (Cimex lectularius, Cimex boueti, Cimex rotundatus, Cimex herundinis) et chez deux acariens (Ornithodorus moubata et Ornithodorus savignyi). Uribe (1926) signale l'infection d'un hémiptère prédateur (Apiomerus pelipes). Rodhain et Brutsaert (1923) obtiennent l'évolution complète chez un mélophage (Melophagus ovinus).

La transmission mécanique, sinon l'évolution, a été démontrée par Neiva (1913) pour deux Ixodes: Rhipicephalus sanguineus et Amblyomma cayennense. Le rôle de la mouche a été mis en évidence par Vergani (1952), puis Carlos Diaz Ungria (1966). D'après Wood (1943), Trypanosoma cruzi peut survivre dans la cavité générale des Réduvidés plus de 127 jours après leur mort, assurant par ingestion l'infestation des mammifères.

En ce qui concerne plus particulièrement les Ornithodores, outre les expériences d'E. Brumpt déjà mentionnées, Mayer et Rocha Lima (1918) ont signalé la longue persistance (cinq ans) de l'infection à $T$. cruzi avec conservation de la virulence chez O. moubata, mais l'impossibilité pour les Ornithodores de transmettre l'infection par piqûre et à leur descendance. Dunn (1934) a montré que l'évolution et la conservation de la virulence étaient possibles chez $O$. venezuelensis pendant au moins six mois, mais qu'elles n'étaient pas possibles ou en tout cas très éphémères chez Ornithodorus talaje. De Beaurepaire Arago, par contre, ne peut faire évoluer $T$. cruzi ni chez $O$. brasiliensis ni chez O. rostratus. Mazzotti et Osorio (1943) conservent une souche de $T$. cruzi virulente 429 jours chez $O$. furcosus, 158 jours sur $O$. parkeri et $O$. amblus; ils obtiennent par contre deux échecs avec $O$. hermsi.

En nous basant sur ces faits acquis, nous avons étudié l'évolution de $T$. cruzi (souche Tulahuen) sur l'Ornithodore (Ornithodorus moubata) dont nous assurons l'élevage dans notre laboratoire.

Par ailleurs, nous avons voulu savoir si certains facteurs, nombre et espacement des repas, développement d'une infection associée, étaient susceptibles d'influer sur la biologie du Trypanosome et si en particulier une souche atténuée par six mois de culture pouvait récupérer sa virulence en repassant par le tube digestif de l'Ornithodore. 


\section{I. - Evolution de Trypanosoma cruzi (Souche Tulahuen) dans le tube digestif d'Ornitbodorus moubata}

Des Ornithodores (Ornithodorus moubata), nourris sur souris infectées à Trypanosoma cruzi (souche Tulahuen), ont été ensuite ponctionnés au niveau du tube digestif ou sacrifiés afin :

- de réaliser des frottis permettant une étude morphologique des divers stades évolutifs des Trypanosomes dans le tube digestif de l'Ornithodore ;

- d'inoculer des animaux pour vérifier l'éventuelle pathogénicité des différentes phases évolutives.

En raison de la conformation du tube digestif de l'Ornithodore (fig. 1), il n'est pas possible, comme chez les Réduvidés, de suivre avec précision le trajet suivi par les Try-

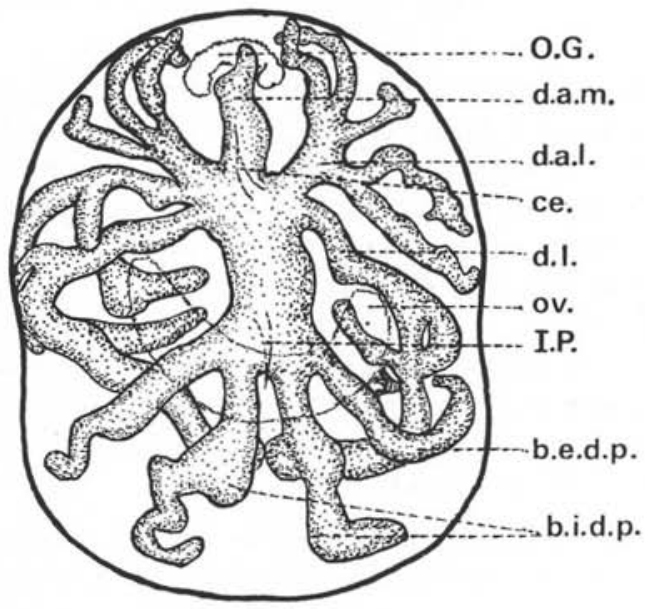

Fig. 1. - Appareil digestif de l'Ornithodorus moubata (cf. Patton et Cragg 1913)

O.G. : organe génital.

d.a.m. : diventricule antéro-médian.

d.a.l. : diventricule antéro-latéral.

oe. : œsophage.

d.l. : diventricule latéral.

ov. : ovaire.

i.p. : intestin postérieur.

b.e.d.p.: branche externe du diventricule postérieur.

b.i.d.p.: branche interne du diventricule postérieur. 
panosomes à partir de leur ingestion jusqu’à leur élimination dans les déjections. Nous avons donc procédé par ponctions aveugles au niveau des différents diverticules du tube digestif ou par broyats.
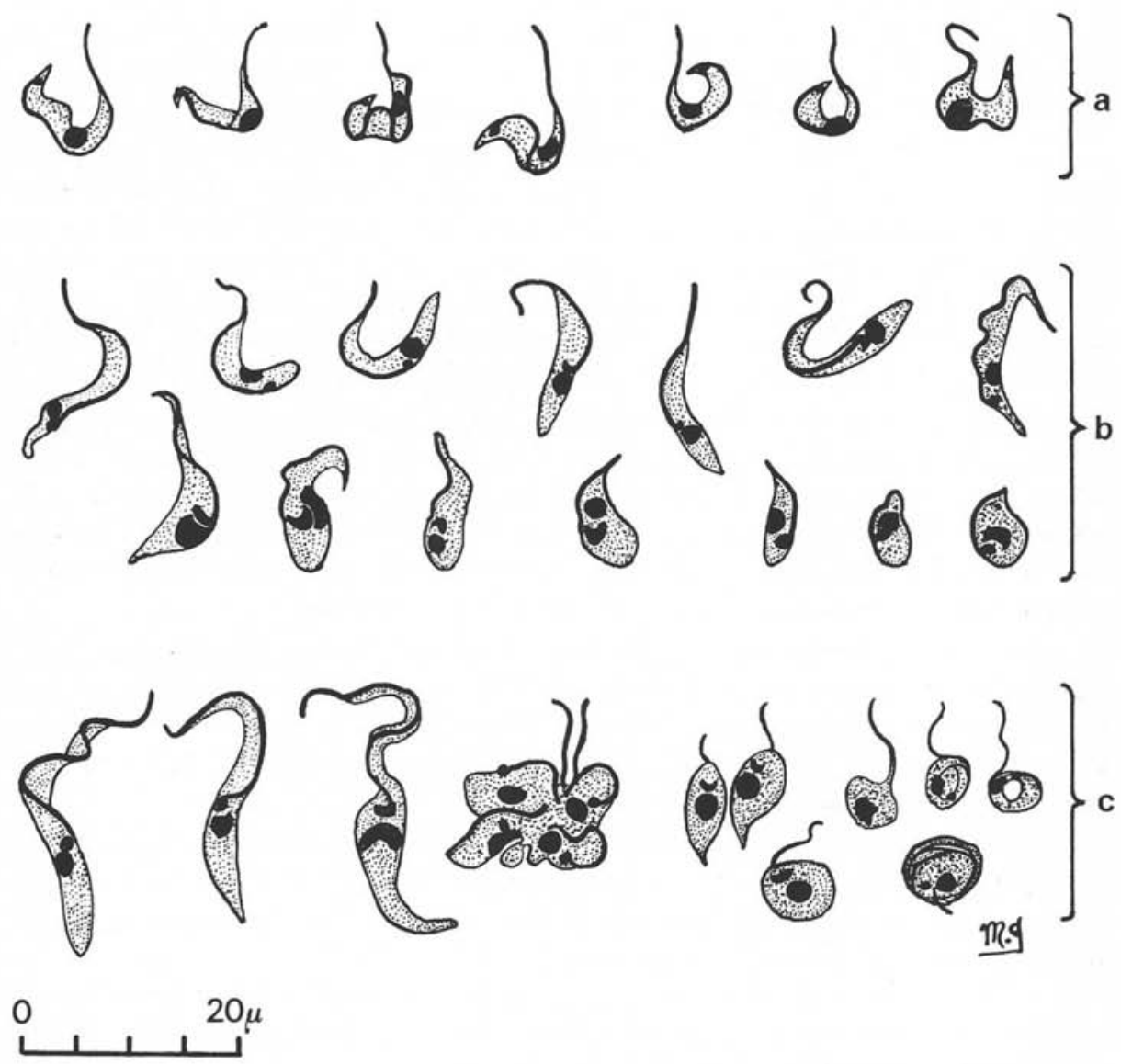

F1G. 2. $-T$. cruzi (Tulahuen) dans le tube digestif d'O. moubata. Formes évolutives de la $24^{\circ}$ heure

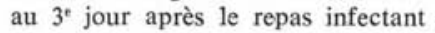

a) formes sanguicoles ingérées lors du repas;

b) formes observées à la $24^{\circ}$ heure ;

c) formes observées les $2^{e}$ et $3^{e}$ jours. Noter la présence des formes caractéristiques de trypanosomes (enroulées ou en voie de dégénérescence). 
A) Etude MorphologiQue.

Dès les premières heures qui suivent l'ingestion des Trypanosomes par l'Ornithodore, on assiste à une transformation morphologique des Trypanosomes en une forme arrondie, trapue. Ce processus, signalé chez les Réduvidés par les différents auteurs qui ont étudié le cycle du Trypanosoma cruzi (Chagas 1909, E. Brumpt 1912, Mayer et Da Rocha Lima 1914, Nino 1928, Dias 1934, Ruge et Rocfer 1957), est considéré comme une forme de régression nécessaire à une future multiplication du parasite au sujet de laquelle les interprétations diffèrent quelque peu: passage initial par le stade leishmania pour les uns, transformation directe en crithidia pour les autres.

Sur les frottis effectués à la $24^{\circ}$ heure, nous notons la persistance de Trypanosomes ayant toutefois subi des modifications morphologiques (migration du blépharoplaste vers le noyau, tendance à la perte du flagelle libre, diminution de la taille). Les formes crithidia typiques sont nombreuses, se multipliant activement, souvent amassées en tas. Il existe par ailleurs des éléments arrondis « leishmaniformes », mesurant de 7 à $10 \mathrm{~m} \mu$, dont il est difficile de préciser s'il s'agit de véritables formes évolutives correspondant à un stade leishmania, ou de Trypanosomes morphologiquement modifiés (fig. 2-b).

Sur les frottis observés les deuxième et troisième jours (fig. 2-c), on retrouve les formes crithidia typiques mesurant en moyenne $32,5 \mathrm{~m} \mu$ et certaines formes géantes jusqu'à 42,5 $\mathrm{m} \mu$. On observe également des formes crithidiennes plus courtes que les précédentes, trapues, « en torpille » et des éléments «leishmaniformes ». Enfin, on note la présence d'éléments arrondis présentant un enroulement intérieur du flagelle et d'éléments évoquant l'enroulement de Trypanosomes sur eux-mêmes.

A partir du cinquième jour (fig. 3) apparaissent les premiers Trypanosomes métacycliques (fig. 3-a) avec tous les intermédiaires de passage du stade Crithidia au stade Trypanosome. Il s'agit de Trypanosomes plus minces et plus longs ( 25 à $28,75 \mathrm{~m} \mu)$ que les formes sanguicoles adultes $(17$ à $22,5 \mathrm{m \mu})$ et dont le kinétoplaste est plus éloigné de l'extrémité postérieure et celle-ci moins pointue.

Les jours suivants, la taille des Trypanosomes métacycliques diminue: 22,5 à $26,1 \mathrm{~m} \mu$, au huitième jour, 22,5 à $25 \mathrm{~m} \mu$, au dixième jour. A côté de ces Trypanosomes métacycliques, on continue à observer, jusqu'au dixième jour, une prolifération des formes crithidias d'aspect et de taille variables $(22,5$ à $52,5 \mathrm{m \mu})$, et d'autre part ces formes si curieuses dont nous avons déjà mentionné l'existence sur les frottis du deuxième et troisième jour et qui nous semblent être des formes de résistance de Trypanosomes (Trypanosomes « d'attente ») (fig. 4).

\section{B) Etude DU POUVOIR INFECTANT DES DIFFÉRENTES Formes ÉVOlutives.}

L'inoculation des broyats d'Ornithodores sacrifiés de jour en jour, dès le premier jour et jusqu'au $30^{\circ}$ jour après leur repas infectant, a permis de constater que tous les broyats étaient infectieux, déterminant chez la souris une infection après une incubation variant de 7 à 32 jours. 


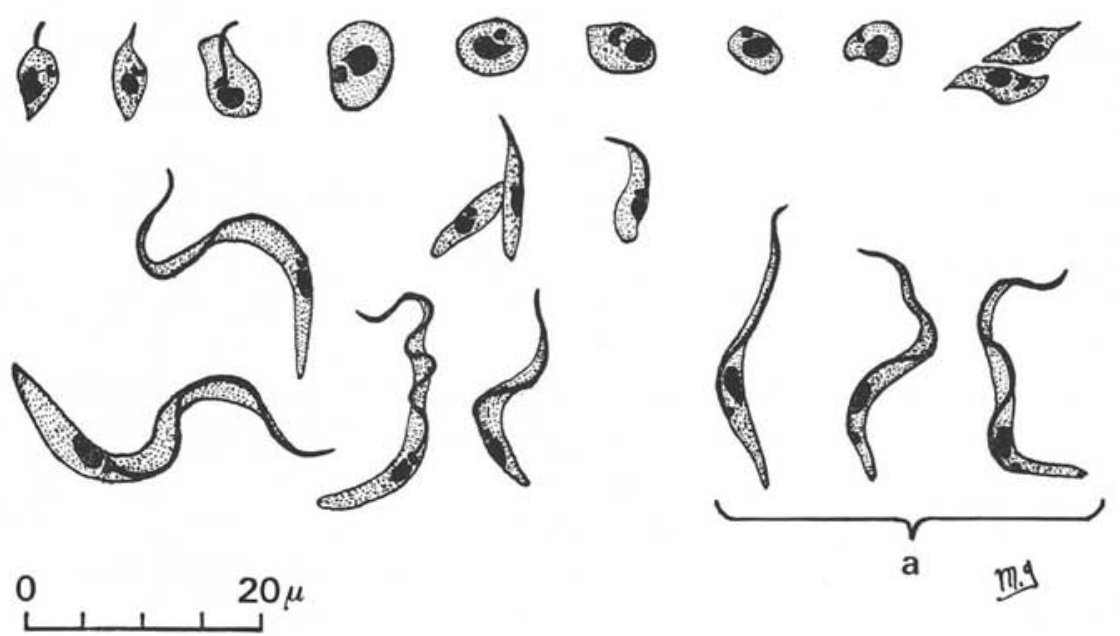

Fig. 3. - T. cruzi (Tulahuen) dans le tube digestif d'O. moubata le $5^{\circ}$ jour après le repas infectant. Apparition des trypanosomes métacycliques (groupe a)
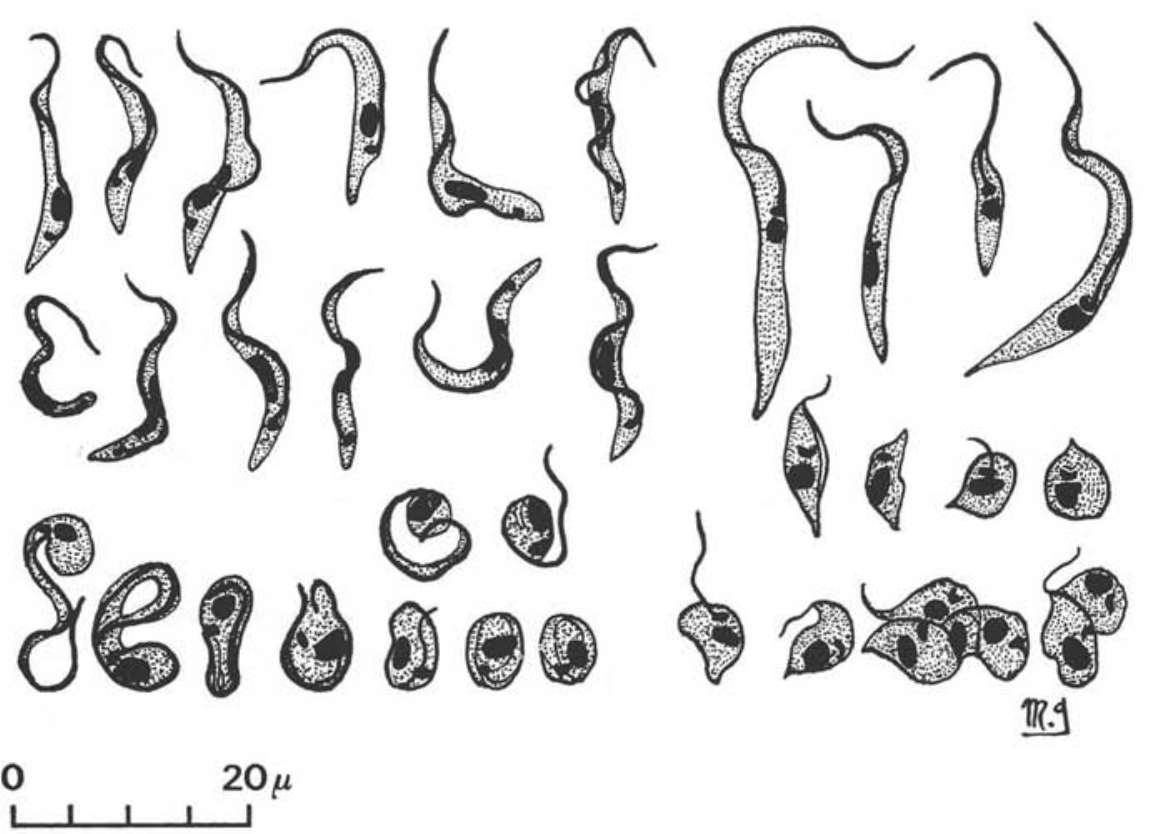

M..

Fig. 4. - T. cruzi (Tulahuen) dans le tube digestif d'O. moubata plus de 5 jours après le repas infectant. Différents aspects évolutifs. Noter la persistance des formes d'enroulement 


\begin{tabular}{|c|c|c|c|c|c|c|c|c|c|c|c|c|c|}
\hline $\begin{array}{l}\text { Dates (en jours) } \\
\text { d'inoculation des } \\
\text { broyats d'Ornitho- } \\
\text { dores après le repas } \\
\text { infectant ........... }\end{array}$ & 1 & 2 & 3 & 4 & 5 & 6 & 7 & 8 & 9 & 10 & 15 & 20 & 30 \\
\hline $\begin{array}{l}\text { Infestation de la } \\
\text { souris. Délais d'in- } \\
\text { cubation (en jours) }\end{array}$ & 11 & 11 & 12 & 13 & 18 & 32 & 10 & 7 & 13 & 10 & 7 & 15 & 10 \\
\hline
\end{tabular}

Le fait que les inoculations antérieures au cinquième jour correspondant à l'apparition des formes Trypanosomes métacycliques (voir à étude morphologique et fig. 3-a) se soient avérées infectantes, peut s'expliquer de deux façons:

- Pouvoir infectant des formes crithidia ;

- Survivance des Trypanosomes ingérés, sous un aspect morphologique dégradé ou de résistance, avec conservation de leur pouvoir pathogène.

En ce qui concerne la première hypothèse, la notion classique admise veut que seuls les Trypanosomes métacycliques soient infectants à l'encontre des autres formes évolutives : «Les flagellés du type crithidia ne sont pas infectieux (E. Brumpt).

A propos du deuxième point, tous les auteurs, à l'exception de Dias (1934), sont d'accord pour affirmer la disparition rapide des Trypanosomes dans le tube digestif de l'insecte et leur transformation, soit d'abord en forme leishmania, soit directement en forme crithidia de multiplication. Il ne semble pas, par contre, que des inoculations du contenu de tubes digestifs des Réduvidés ou leur broyat, dans les jours immédiats, qui suivent le repas infectant et avant l'apparition des formes métacycliques infectieuses, aient été réalisées.

Mais le problème a été mieux étudié en culture où les aspects morphologiques reproduits sont identiques à ceux produits chez l'insecte vecteur. En effet, lorsqu'on ensemence le sang de vertébré infecté dans un milieu quelconque, les flagellés passent par les stades Leishmania, Leptomonas et Trypanosome. Galliard (1929) a montré que l'évolution était d'ailleurs variable suivant le milieu employé. Avec des milieux gélosés semi-solides, cet auteur ne trouve jamais de forme leishmania ou leptomonas; les formes crithidia se multiplient activement aussitôt ensemencées et pullulent déjà au bout de 3 à 4 jours sans former de rosaces, contrairement à ce qui se passe dans le milieu N.N.N. Le problème important est de savoir dans quelles conditions et à quel moment apparaissent les Trypanosomes. A ce propos, les avis diffèrent ; Delanoe (1912) n'en a jamais retrouvé, Torrès (1922) les retrouve d'une façon inconstante, Muniz (1927) constate leur apparition au bout de 15 jours, Nino (1929) beaucoup plus tardivement (76 $6^{e}$ jour). La température, d'après Noller (1920), exercerait une action prépondérante (optimum $30^{\circ}$ ). Par contre, quel que soit le milieu employé et à la température ordinaire $\left(25^{\circ}\right)$, Galliard observe leur apparition d'une façon extrêmement précoce et particulièrement en milieu gélosé sucré ( $5^{\circ}$ jour), en tenant compte toutefois « de la possibilité d'une survivance possible quoiqu'invraisemblable des formes ensemencées ». 


\title{
II. - Conservation de la virulence d'une souche
} de Trypanosoma cruzi (tulabuen) chez O. moubata

\author{
Etude des facteurs susceptibles de modifier la biologie \\ du Trypanosome dans l'intestin de l'Ornithodore
}

A) OrNithodores adUltes N'Ayant EFFectú́ QU'Un SEUL REPAS SUR LA SOURIS (REPAS INFECTANT).

Ce repas a été effectué sur une souris infectée depuis 15 jours avec la souche Tulahuen de Trypanosoma cruzi et présentant environ $4 \mathrm{Tr} / \times$. Dans des délais de 3, 6, 13, 21, 34, 111 jours, des formes évolutives de Trypanosoma cruzi ont été retrouvées dans le liquide de ponction cæcal des Ornithodores et l'inoculation à la souris du contenu cæcal ou du broyat des Ornithodores a toujours déterminé une infection mortelle dans des délais de 21 à 70 jours (délais de survie des souris témoins, moyenne: 17 jours).

L'expérience n'a pas été poursuivie régulièrement au-delà du $111^{\circ}$ jour, mais chez des Ornithodores à jeun depuis deux ans après le seul repas infectant, la persistance de l'infection n'a pu être décelée.

B) ORNITHOdores AYANT ÉtÉ RÉGULIÈrEMENT NOURRIS SUR SOURIS NON INFECTÉES APRÈS LE PREMIER REPAS INFECTANT.

Chez des Ornithodores ayant reçu un ou plusieurs repas à des dates différentes après le repas infectant, des formes évolutives de Trypanosoma cruzi ont toujours été décelées en plus ou moins grand nombre dans la ponction de liquide cæcal ou le broyat des Ornithodores aux dates suivantes $\left(32^{\circ}, 72^{\circ}, 115^{\circ}, 156^{\circ}, 163^{\circ}, 221^{\circ}, 326^{\circ}, 341^{\circ}, 344^{\circ}\right.$, $352^{\circ}, 430^{\circ}, 434^{\circ}, 442^{\circ}, 632^{\circ}, 640^{\circ}, 695^{\circ}, 791^{\circ}$ jour) et l'inoculation à la souris a toujours déterminé une infection.

Chez des Ornithodores qui semblaient avoir perdu l'infection (opinion basée sur l'absence de formes évolutives de Trypanosomes dans la petite quantité de liquide cæcal recueilli par ponction), après un seul repas infectant et cesi respectivement au $147^{\circ}$ jour (deux exemplaires), $155^{\circ}$ jour (trois exemplaires), $231^{\circ}$ jour, $325^{\circ}$ jour, $327^{\circ}$ jour (deux exemplaires), l'infection a été « réactivée " par un repas sur souris neuve puisque, dans tous les cas, l'examen de la ponction du liquide cæcal alors plus abondante a permis d'observer des parasites en grande quantité dans des délais de 5 à 47 jours suivant le deuxième repas.

En résumé, il nous est donc permis d'affirmer que l'infection du tube digestif de l'Ornithodore s'est maintenue pendant 791 jours. Il ne s'agit pas là d'ailleurs d'un « record », puisque Mayer et Da Rocha Lima (1914) ont mentionné la persistance de l'infection pendant cinq ans chez Ornithodorus moubata. 


\section{III. - Essai de transmission de l'infection par piqûre d'Ornithodores infectés et par inoculation de broyats de leurs larves à des animaux neufs}

Sur un total de huit lots comportant 28 Ornithodores infectés depuis 32 à 328 jours, les essais de transmission par piqûre ont toujours été négatifs, ce qui confirme les expériences antérieures de Mayer et Da Rocha Lima (1918), de même que les inoculations de broyats de pontes et de larves provenant de parents infectés.

\section{IV. - Essai d'exaltation de la virulence de Trypanosoma cruzi (tulabuen)}

\section{atténuée par culture par passage sur Ornithodorus moubata Etude comparée par passage sur Rbodnius prolixus}

A) UN LOT DE 14 RhodniUs a été nourri sur des souris respectivement infectées à partir de cultures, depuis six mois à un an et présentant une infection chronique. L'inoculation à des souris neuves des déjections infectées de Rhodnius ou du broyat de ces mêmes Rhodnius dans des délais de 10, 25, 110, 146, 178 et 229 jours, après le repas infectant, a toujours déterminé chez la souris une infection évoluant sur le mode chronique avec parasitémie maximum de un Trypanosome par champ.

Les passages en série de souris à souris, effectués à partir de ces infections chroniques, obtenues par déjections de Rhodnius, ne nous ont pas permis d'obtenir une exaltation de la virulence de la souche, se traduisant par des infections mortelles. L'emploi de la Cortisone et des injections de sérum glucosé s'est avéré sans efficacité.

B) UN LOT DE 27 ORNITHOdoRus moubata a été nourri sur des souris respectivement infectées à partir de culture depuis six mois à un an, ayant toutes fait une infection chronique. Au moment du repas, la parasitémie était faible $(1 \mathrm{Tr} / 50 \times$ grossissement 250).

Les broyats de ces Ornithodores, effectués ultérieurement dans des délais de 25, $57,61,68,146,168$ jours après le repas infectant et dont l'examen microscopique a permis de constater la présence de formes évolutives de Trypanosoma cruzi (17 fois sur 27) ont été inoculés à des souris neuves. Sur les 27 souris, trois seulement sont décédées d'une infection aiguë du $24^{\circ}$ au $32^{\circ}$ jour $(20 \mathrm{~T} / \times)$, correspondant aux broyats d'Ornithodores effectués 25 jours après le repas infectant. Deux autres ne se sont jamais infectées, correspondant à des Ornithodores nourris sur des souris infectées depuis 168 jours (donc à jeun) et dans les broyats desquels l'examen microscopique n'avait pas 
permis de retrouver de forme évolutive de $T$. cruzi. Dans les 22 autres cas, toutes les souris ont fait des infections chroniques bénignes et le passage en série à des souris neuves (six passages en un an) n'a pas permis d'obtenir d'exaltation de la virulence même chez les souris soumises à un traitement par la Cortisone ou à des injections répétées de sérum glucosé.

En résumé, le passage sur Ornithodorus moubata de la souche Tulahuen de Trypanosoma cruzi atténuée par culture nous a permis d'obtenir une exaltation de la virulence trois fois sur 27 essais. Sans vouloir établir de comparaison définitive, ce phénomène paraît d'autant plus intéressant à signaler que la même expérience faite en parallèle sur un lot, il est vrai moins important de Rhodnius (14 exemplaires), s'est soldé par un échec.

\section{V. - Etude du développement de l'infection simultanée à Trypanosoma cruzi et Borrelia duttoni (souche Congo) chez l'Ornithodore}

En fonction des phénomènes protecteurs de l'infection borrélienne à l'égard de certaines trypanosomoses chez la souris et en particulier de Trypanosoma cruzi, il était intéressant d'étudier chez l'Ornithodore les possibilités de développement simultané de l'infection à Borrelia duttoni (Congo) et Trypanosoma cruzi.

Un premier lot d'Ornithodores a d'abord été infesté avec Borrelia duttoni, puis, 45 jours plus tard, par Trypanosoma cruzi (Tulahuen). Par piqûre, les Ornithodores ont continué à transmettre normalement et seulement l'infection borrélienne, tandis que l'inoculation des broyats n'assurait la double infection qu'irrégulièrement. Par trois fois, en effet, les souris neuves inoculées ne s'infectèrent pas en Trypanosomes.

Ces échecs doivent s'interpréter en fonction des phénomènes protecteurs connus de l'infection borrélienne à l'égard des Trypanosomes chez la souris. Mais, chez l'Ornithodore, la coexistence de l'infection à Trypanosoma cruzi et Borrelia duttoni est parfaitement possible.

\section{Bibliographie}

[Voir à l'article suivant: Evolution de T. cruzi (Tchuantepec) chez Triatoma megista]. 